\title{
Impulsive Consensus for Leader-Following Multiagent Systems with Fixed and Switching Topology
}

\author{
Zhi-Wei Liu, ${ }^{1,2}$ Zhi-Hong Guan, ${ }^{2}$ and Hong Zhou ${ }^{1}$ \\ ${ }^{1}$ Department of Automation, Wuhan University, Wuhan 430072, China \\ ${ }^{2}$ College of Automation, Huazhong University of Science and Technology, Wuhan 430074, China \\ Correspondence should be addressed to Zhi-Hong Guan; zhguan@mail.hust.edu.cn
}

Received 29 March 2013; Accepted 2 June 2013

Academic Editor: Chuandong Li

Copyright (c) 2013 Zhi-Wei Liu et al. This is an open access article distributed under the Creative Commons Attribution License, which permits unrestricted use, distribution, and reproduction in any medium, provided the original work is properly cited.

\begin{abstract}
This paper studied the consensus problem of the leader-following multiagent system. It is assumed that the state information of the leader is only available to a subset of followers, while the communication among agents occurs at sampling instant. To achieve leaderfollowing consensus, a class of distributed impulsive control based on sampling information is proposed. By using the stability theory of impulsive systems, algebraic graph theory, and stochastic matrices theory, a necessary and sufficient condition for fixed topology and sufficient condition for switching topology are obtained to guarantee the leader-following consensus of the multiagent system. It is found that leader-following consensus is critically dependent on the sampling period, control gains, and interaction graph. Finally, two numerical examples are given to illustrate the effectiveness of the proposed approach and the correctness of theoretical analysis.
\end{abstract}

\section{Introduction}

During the past several decades, the consensus problem of the multiagent system has drawn a great deal of attentions because of its broad applications in many domains, including distributed coordination [1], synchronization of dynamical networks [2], distributed filtering [3], and load balancing [4]. The basic idea of consensus is to design a distributed control such that the team of agents can achieve a state agreement only by locally available information without central control stations. Consensus problem has been addressed in various situations, such as time delay [5], switching topology [6], asynchronous algorithms $[4,7]$, nonlinear algorithms $[8,9]$, quantized data $[4,10]$, noisy communication channel [11], and second-order model $[12,13]$.

Inspired by some biological systems and engineering applications, the leader-following consensus problem has received a lot of interest. The leader is a special agent whose motion is independent of all other agents and thus is followed by all other agents. It has been widely used in many applications $[14,15]$. For the first-order multiagent systems, Jadbabaie et al. [16] considered a leader-following consensus problem and discussed the convergence properties of the leader-follower systems. Cao and Ren [17] studied a leaderfollowing consensus problem with reduced interaction for both first- and second-order multiagent systems. Su et al. [18] studied a flocking algorithm with a virtual leader. Zhu and Cheng [19] considered leader-following consensus of secondorder agents with multiple time-varying delays. Meng et al. [20] studied the leaderless and leader-following consensus algorithms with communication and input delays under a directed network by the Lyapunov theorems and the Nyquist stability criterion.

In recent years, owing to the development of digital sensors and the constraints of transmission bandwidth of networks, many control systems can be modeled by continuoustime systems together with discrete sampling. Therefore, it is significant to design the distributed control for continuoustime multiagent systems based on sampled information. There are a few reports [20-25] dealing with this problem, where the control inputs regulate the velocity of each agent continuously over the sampling period.

On the other hand, impulsive dynamical systems exhibit continuous evolutions typically described by ordinary differential equations and instantaneous state jumps or impulses. 
It is also well known that the impulsive control is more efficient than one of continuous control in many situations. The examples include ecosystems management [26], orbital transfer of satellite [27], and optimal control of economic systems [28]. The main idea of impulsive control is to instantaneously change the state of a system when some conditions are satisfied. During the last few decades, it has been widely applied into the synchronization problems of complex dynamical networks [29-31], which can be regarded as first-order multiagent systems with nonlinear dynamics. In many real-world system, agents are governed by both position and velocity dynamics. The impulsive control for second-order multiagent system was studied in [32, 33], where both velocity and position are instantaneously changed by impulsive control, but position cannot change quickly in many situation. Therefore, it is more reasonable to only regulate the velocity of each agent to reach consensus [34, 35]. In [34], we designed impulsive velocity-control for multiagent systems with fixed topology to achieve consensus. In [35], an impulsive control was proposed in which the current position data of its neighbours and the past position data of its own state were utilised to regulate the velocity of agents.

This paper aims to investigate the consensus problem of leader-following multiagent systems by using impulsive control which only regulates the velocity of agents. Our main contributions are summarised as follows. First, a necessary and sufficient condition under fixed topology is derived, and it is found that the leader-following consensus in multiagent systems with sampling information can be reached if and only if the sampled period is bounded by critical values which depend on control gains and the interaction graph. Second, a sufficient condition under switching topology is obtained, and it is shown that the impulsive interval is restricted by an upper bound which depends on control gains, the diagonal element of the Laplacian matrix, and the connections between agents and leader. The two key difference between this paper and our earlier work [34] are that the leader-following case is taken into account and that this paper considers multiagent systems under switching topology.

The remainingpart of the paper is organized as follows. In Section 2, some necessary mathematical preliminaries are given. Main results of this paper, that is, the convergence of the distributed impulsive control under fixed and switching topology, are presented in Sections 3 and 4. In Section 5, some illustrative numerical examples are given. Concluding remarks are finally stated in Section 6.

\section{Problem Formulation}

Let $\mathbb{R}$ and $\mathbb{C}$ denote the set of real numbers and complex numbers, respectively. For $A=\left(a_{i j}\right)_{m \times m} \in \mathbb{R}^{m \times m}, \lambda_{1}(A)$, $\lambda_{2}(A), \ldots, \lambda_{m}(A)$ are the eigenvalues of $A, \rho(A)$ represent the spectral radius of $A$. The identity matrix of order $n$ is denoted as $I_{n}$ (or simply $I$ if no confusion arises). For $\gamma \in \mathbb{C}, \operatorname{Re}(\gamma)$ and $\operatorname{Im}(\gamma)$ are the real and imaginary part of $\gamma$, respectively. $\mathbf{1}_{n}=(1,1, \ldots, 1)^{T}$ is the column vector. $\mathbf{0}_{n \times m}$ denotes the $n \times m$ matrix with all elements equal to zero.
Let $\mathscr{G}=\{\mathscr{V}, \mathscr{E}, \mathscr{A}\}$ be a directed graph (digraph) with the set of nodes $\mathscr{V}=\{1,2, \ldots N\}$, the set of edges $\mathscr{E} \in \mathscr{V} \times \mathscr{V}$, and the weighted adjacency matrix $\mathscr{A}=\left(a_{i j}\right)_{N \times N}$. In the digraph $\mathscr{G}$, node $i$ represents the agent $i$, and an edge in $\mathscr{G}$ is denoted by an ordered pair $\{j, i\} .\{j, i\} \in \mathscr{E}$ if and only if the agent $i$ can directly receive information from the $j$ th agent. In this case, the $j$ th agent is the neighbor of the $i$ th agent. The set of neighbors of the $i$ th agent is denoted by $\mathscr{N}_{i}=\{j \in \mathscr{V} \mid(j, i) \in \mathscr{E}\}$. All elements of adjacency matrix are nonnegative. For $i, j \in \mathscr{V}, j \in \mathcal{N}_{i} \Leftrightarrow a_{i j}>0$, and assume that $a_{i i}=0, i \in \mathscr{V}$. A directed path in a digraph $\mathscr{G}$ is an ordered sequence $v_{1}, v_{2}, \ldots, v_{k}$ of agents such that any ordered pair of vertices appearing consecutively in the sequence is an edge of the digraph, that is, $\left(v_{i}, v_{i+1}\right) \in \mathscr{E}$, for any $i=1,2, \ldots, k-1$. A directed tree is a digraph, where there exists an agent, called the root, such that any other agent of the digraph can be reached by one and only one path starting at the root. $\mathscr{T}_{\mathscr{G}}=\left\{\mathscr{V}_{\mathscr{T}}, \mathscr{E}_{\mathscr{T}}\right\}$ is a directed spanning tree of $\mathscr{G}$, if $\mathscr{T}_{\mathscr{G}}$ is a directed tree and $\mathscr{V}_{\mathscr{T}}=\mathscr{V}$. The Laplacian matrix $L(\mathscr{G})=\left(l_{i j}\right)_{N \times N}$ of $\mathscr{G}$ is defined as

$$
l_{i j}= \begin{cases}-a_{i j}, & i \neq j, \\ \sum_{k=1, k \neq i}^{N} a_{i k,} & i=j .\end{cases}
$$

Given a matrix $\mathbf{P}=\left(p_{i j}\right) \in \mathbb{R}^{N \times N}$, the digraph (without self-link) of $\mathbf{P}$ denotes by $\mathscr{G}(\mathbf{P})$, which is the digraph with node set $\mathscr{V}=\{1,2, \ldots, N\}$ such that there is an edge in $\mathscr{G}(\mathbf{P})$ from $j$ to $i$ if and only if $p_{i j} \neq 0$. The matrix $A$ is nonnegative, that is, $A \geq 0$, if all element of $A$ is non-negative. The matrix $A, B \in \mathbb{R}^{N \times N}, A \geq B$ denote $A-B \geq 0$. The nonnegative matrix $A$ is row stochastic if all of its row sum are equal to 1 . The row stochastic matrix $A \in \mathbb{R}^{N \times N}$ is called indecomposable and aperiodic (SIA) if $\lim _{k \rightarrow \infty} A^{k}=\mathbf{1}_{N} y^{T}$, where $y$ is some $N \times 1$ column vector.

Consider that a multiagent system consists of $N$ identical agents indexed by $1,2, \ldots, N$, which is described by

$$
\dot{x}_{i}(t)=v_{i}(t), \quad \dot{v}_{i}(t)=u_{i}(t),
$$

where $i=1,2, \ldots, N, x_{i}(t) \in \mathbb{R}, v_{i}(t) \in \mathbb{R}$ are the position and velocity states of the agent $i$, respectively. $u_{i}(t) \in \mathbb{R}^{n}$ is a control input for $i=1,2, \ldots, N$. The static leader for the system (2) is a static agent represented by $x_{0}(t)=x_{0}$, where $x_{0} \in \mathbb{R}$. The edges between the agents and the leader is unidirectional; namely, there are only partial agents that can obtain information from the leader. It is also assumed that each agent can only obtain information from other agents or the leader at sampling times.

This paper focuses on the problem of designing $u_{i}(t)$, $i=1,2, \ldots, N$ based on sampling information to make all $\mathrm{N}$ agents converge to a static leader.

Definition 1. The leader-following consensus of the multiagent system (2) with static leader is said to be achieved if

$$
\lim _{t \rightarrow+\infty} x_{i}(t)=x_{0}, \quad \lim _{t \rightarrow+\infty} v_{i}(t)=0, \quad i \in \mathscr{V},
$$

for any initial state. 


\section{Leader-Following Consensus under Fixed Topology}

In this section, the leader-following consensus problem under fixed topology is considered. The interaction between agents in this part is described by a fixed digraph $\mathscr{G}=$ $\{\mathscr{V}, \mathscr{E}, \mathscr{A}\}$, and the connections between agents and leader are described by $b_{i} \in \mathbb{R}, b_{i}>0$ if and only if the agent $i$ can obtain information from the leader, otherwise, $b_{i}=0$.

In order to achieve the leader-following consensus of the multiagent system (2) with sampled information under fixed topology, the impulsive control for the agent $i$, is designed as

$$
\begin{aligned}
u_{i}(t)=-\sum_{k=1}^{+\infty} p_{1}\left[\left(\sum_{j \in N_{i}} l_{i j}\left(x_{j}(t)-x_{i}(t)\right)\right.\right. \\
\left.\left.\quad+b_{i}\left(x_{i}(t)-x_{0}\right)\right)+p_{2} v_{i}(t)\right] \delta\left(t-t_{k}\right),
\end{aligned}
$$

where $i \in \mathscr{V}$, the sampling time sequence $\left\{t_{k}\right\}_{k=1}^{\infty}$ satisfies $t_{k+1}-t_{k}=h\left(h \in \mathbb{R}\right.$ is sampled period) and $\lim _{k \rightarrow \infty} t_{k}=\infty$, $p_{1}, p_{2}>0$ are the control gain to be determined, and $\delta(\cdot)$ is the Dirac impulsive function.

Equivalently, the multiagent system (2) with impulsive controller (4) can be rewritten as follows:

$$
\begin{gathered}
\dot{x}_{i}(t)=v_{i}(t), \quad \dot{v}_{i}(t)=0, \quad t \in\left(t_{k}, t_{k+1}\right], \\
\Delta v_{i}\left(t_{k}\right)=-p_{1}\left(\sum_{j \in \mathscr{V}} l_{i j} x_{j}\left(t_{k}\right)+b_{i}\left(x_{i}\left(t_{k}\right)-x_{0}\right)\right)-p_{2} v_{i}\left(t_{k}\right),
\end{gathered}
$$

where $\Delta v_{i}\left(t_{k}\right)=v_{i}\left(t_{k}^{+}\right)-v_{i}\left(t_{k}\right), v_{i}\left(t_{k}^{+}\right)=\lim _{t \rightarrow t_{k}^{+}} v_{i}\left(t_{k}\right)$. For simplicity, it is assume that $v_{i}(t)$ is left continuous at $t_{k}$.

Remark 2. From (5), the control input of each agent only uses the information from its neighbors at sampling instants and are only applied at sampling instants. This is quite different from the previously mentioned works, where the control inputs are applied continuously. The velocity of the agent is instantaneously changed at sampling times. This is feasible when the operating time of the impulsive controller is much smaller than the sampled period.

Lemma 3. The multiagent system (2) with impulsive control (4) achieves leader-following consensus asymptotically if and only if $\rho(P)<1$, where

$$
\begin{gathered}
P=\left(\begin{array}{ccc}
I_{N} & & h I_{N} \\
-p_{1}(L+B) & \left(1-p_{2}\right) I_{N}-p_{1} h(L+B)
\end{array}\right), \\
B=\left(\begin{array}{llll}
b_{1} & & & \\
& b_{2} & & \\
& & \ddots & \\
& & & b_{N}
\end{array}\right) .
\end{gathered}
$$

Proof. Let $\hat{x}_{i}(t)=x_{i}(t)-x_{0}(t)$, for $i \in \mathscr{V}$ and note that $\sum_{j \in \mathscr{V}} l_{i j} x_{0}=0$, system (5) can be rewritten as follows:

$$
\begin{aligned}
\dot{\hat{x}}_{i}(t) & =v_{i}(t), \quad \dot{v}_{i}(t)=0, \quad t \in\left(t_{k}, t_{k+1}\right], \\
\Delta v_{i}\left(t_{k}\right) & =-p_{1}\left(\sum_{j \in \mathscr{V}} l_{i j} \widehat{x}_{j}\left(t_{k}\right)+b_{i} \widehat{x}_{i}\left(t_{k}\right)\right)-p_{2} v_{i} .
\end{aligned}
$$

From (7), one has

$$
\begin{gathered}
\hat{x}_{i}\left(t_{k+1}\right)=\widehat{x}_{i}\left(t_{k}\right)+h v_{i}\left(t_{k}^{+}\right), \\
v_{i}\left(t_{k+1}\right)=v_{i}\left(t_{k}^{+}\right), \\
v_{i}\left(t_{k+1}^{+}\right)=\left(1-p_{2}\right) v_{i}\left(t_{k+1}\right) \\
-p_{1}\left(\sum_{j \in \mathscr{V}} l_{i j} \widehat{x}_{j}\left(t_{k+1}\right)+b_{i} \widehat{x}_{i}\left(t_{k+1}\right)\right) .
\end{gathered}
$$

Then, the evolution of $\hat{x}_{i}\left(t_{k}\right), v_{i}\left(t_{k}\right)$ under impulsive control (4) can be described as follows:

$$
\begin{aligned}
& \hat{x}_{i}\left(t_{k+1}\right)=\widehat{x}_{i}\left(t_{k}\right)+h v_{i}\left(t_{k}^{+}\right), \\
& v_{i}\left(t_{k+1}^{+}\right)=\left(1-p_{2}\right) v_{i}\left(t_{k}^{+}\right) \\
&-p_{1} h \sum_{j \in \mathscr{V}} l_{i j} v_{j}\left(t_{k}^{+}\right)-p_{1} b_{i} h v_{i}\left(t_{k}^{+}\right) \\
&-p_{1}\left(\sum_{j \in \mathscr{V}} l_{i j}\left(\hat{x}_{j}\left(t_{k}\right)\right)+b_{i} \widehat{x}_{i}\left(t_{k}\right)\right) .
\end{aligned}
$$

Let $\hat{x}(k)=\left(\hat{x}_{1}\left(t_{k}\right), \ldots, \widehat{x}_{N}\left(t_{k}\right)\right)^{T}$ and $v(k)=\left(v_{1}\left(t_{k}^{+}\right), \ldots\right.$, $\left.v_{N}\left(t_{k}^{+}\right)\right)^{T}$. Then, the multiagent system (2) achieves leaderfollowing consensus, if and only if $\lim _{k \rightarrow+\infty} \widehat{x}(k)=0$, $\lim _{k \rightarrow+\infty} v(k)=0$.

Equivalently, (10) can be rewritten as follows:

$$
\left(\begin{array}{l}
\widehat{x}(k+1) \\
v(k+1)
\end{array}\right)=P \times\left(\begin{array}{l}
\widehat{x}(k) \\
v(k)
\end{array}\right) .
$$

Therefore, it is easy to obtain the result by the stability theory of discrete-time systems.

The following lemmas and definition are needed for the subsequent development.

Lemma 4 (bilinear transformation theorem [36]). Polynomial $R(z)$ (of degree $d$ ) is Schur stable if and only if $Q(z)$ is Hurwitz stable, where

$$
R(z)=(z-1)^{d} Q\left(\frac{z+1}{z-1}\right) .
$$


For complex polynomial $R(z)$, let

$$
R(i \omega)=m(\omega)+i n(\omega),
$$

where $m(\omega), n(\omega) \in \mathbb{R}$ and $i$ is the imaginary unit.

Lemma 5 (see $[37,38])$. The complex polynomial $R(z)=z^{2}+$ $a z+b$, where $\alpha \in \mathbb{C}$ and $\beta \in \mathbb{C}$, is Hurwitz stable if and only if $\operatorname{Re}(a)>0$ and $\operatorname{Re}(a) \operatorname{Im}(a) \operatorname{Im}(b)+\operatorname{Re}^{2}(a) \operatorname{Re}^{2}(b)-\operatorname{Im}^{2}(b)>0$.

Next theorem will show what kind of interaction topology can reach leader-following consensus and how to determine the control gains $p_{1}, p_{2}$ and sampling period $h$.

Theorem 6. The multiagent system (2) with impulsive control (4) under fixed topology achieves the leader-following consensus asymptotically if and only if

$$
h<\min _{1<i<N} \frac{2 p_{2}^{2}\left(2-p_{2}\right) \operatorname{Re}\left(\lambda_{i}\right)}{\operatorname{Im}^{2}\left(\lambda_{i}\right) p_{1}\left(p_{2}-2\right)^{2}+\operatorname{Re}^{2}\left(\lambda_{i}\right) p_{1} p_{2}^{2}},
$$

where $\lambda_{i}, i=1,2, \ldots, N$ are the eigenvalues of $L+B$.

Proof. Let the $\gamma$ be an eigenvalue of matrix $P$. Then,

$$
\begin{aligned}
\operatorname{det} & \left(\gamma I_{2 N}-P\right) \\
= & \operatorname{det}\left(\begin{array}{cc}
(\gamma-1) I_{N} & -h I_{N} \\
p_{1}(L+B) & \gamma I_{N}-\left(1-p_{2}\right) I_{N}+p_{1} h(L+B)
\end{array}\right) \\
= & \prod_{i=1}^{N}\left(\gamma^{2}+\gamma\left(p_{2}-2+p_{1} h \lambda_{i}\right)+1-p_{2}\right) .
\end{aligned}
$$

Let

$$
Q_{i}(\gamma)=\gamma^{2}+\gamma\left(p_{2}-2+p_{1} h \lambda_{i}\right)+1-p_{2}, \quad i \in \mathscr{V} .
$$

Then, we only need to prove that polynomials $Q_{i}(\gamma)$ for $i \in \mathscr{V}$ are Schur stable.

Let

$$
\begin{aligned}
R_{i}(\sigma) & =(\sigma-1)^{2} Q_{i}\left(\frac{\sigma+1}{\sigma-1}\right) \\
& =p_{1} h \lambda_{i} \sigma^{2}+2 p_{2} \sigma+4-2 p_{2}-p_{1} h \lambda_{\mathrm{i}} .
\end{aligned}
$$

Let

$$
R^{\prime}(\sigma)=\sigma^{2}+\frac{2 p_{2}}{p_{1} h} \lambda_{i}^{\prime} \sigma+\left(\frac{4}{p_{1} h}-\frac{2 p_{2}}{p_{1} h}\right) \lambda_{i}^{\prime}-1,
$$

where $\lambda_{i}^{\prime}=1 / \lambda_{i}^{\prime}$. Then, according to Lemma 4 , polynomials $R_{i}^{\prime}(\sigma)$, for $i=1,2,3, \ldots, N$, are Hurwitz stable if and only if polynomials $Q_{i}(\gamma)$ for $i=1,2,3, \ldots, N$ are Schur stable.

It can be proved by Lemma 5 that $R_{i}(\sigma)$ is Hurwitz stable if and only if (14) is satisfied. Therefore, $\rho(P)<1$ if and only if (14) is satisfied. The proof is thus completed.

Remark 7. It can be observed from the inequality (14) that the real and imaginary part of the eigenvalues of $L+B$, the sampling period $h$, and two control gains $p_{1}$ and $p_{2}$ play important roles in achieving consensus. $p_{2}<2$ and $\operatorname{Re}\left(\lambda_{i}(L+\right.$ $B))>0$, for $i \in \mathscr{V}$, are necessary conditions for leaderfollowing consensus. It is easy to see that the critical value of $h$ increases as $p_{1}$ decreases.

Remark 8. Let $\widetilde{\mathscr{G}}=\{\widetilde{\mathscr{V}}, \widetilde{\mathscr{E}}, \widetilde{\mathscr{A}}\}$ with $\widetilde{\mathscr{V}}=\{0,1,2, \ldots, N\}$, and the Laplace matrix is

$$
\widetilde{L}=\left(\begin{array}{ccccc}
0 & 0 & 0 & \cdots & 0 \\
-b_{1} & l_{11}+b_{1} & l_{12} & \cdots & l_{1 N} \\
-b_{2} & l_{21} & l_{22}+b_{2} & \cdots & l_{2 N} \\
\vdots & \vdots & \vdots & \ddots & \vdots \\
-b_{N} & l_{N 1} & l_{N 2} & \cdots & l_{N N}+b_{N}
\end{array}\right) .
$$

Note that

$$
E^{-1} \widetilde{L} E=\left(\begin{array}{cc}
0 & \mathbf{0}_{N}^{T} \\
\mathbf{0}_{N} & L+B
\end{array}\right)
$$

where

$$
E=\left(\begin{array}{cc}
1 & \mathbf{0}_{N}^{T} \\
\mathbf{1}_{N} & I_{N}
\end{array}\right)
$$

is an invertible matrix. $\operatorname{Re}\left(\lambda_{i}\right)>0$, for $i \in \mathscr{V}$ imply that $\widetilde{L}$ has a simple eigenvalue 0 , and all the other eigenvalues have positive real parts. This implies that the graph $\widetilde{\mathscr{G}}$ contains a spanning tree. The root of the spanning tree is the leader.

Remark 9. How to choose a suitable control gain $p_{1}$ and $p_{2}$ when the sampling period $h$ is given. According to Theorem $6, p_{2}<2$ is a necessary condition for consensus. Therefore, one can choose $p_{2}$ from $(0,2]$, and then compute

$$
\Theta=\min _{i \in \mathscr{V}} \frac{2 p_{2}^{2}\left(2-p_{2}\right) \operatorname{Re}\left(\lambda_{i}\right)}{\left(\operatorname{Im}\left(\lambda_{i}\right)\left(p_{2}-2\right)\right)^{2} h+h p_{2}^{2} \operatorname{Re}^{2}\left(\lambda_{i}\right)} .
$$

Then, one can choose $p_{1}$ from $(0, \Theta)$.

\section{Leader-Following Consensus under Switching Topology}

In this section, the leader-following consensus under switching topology is considered. The interaction between agents at sampling time $t_{k}$ is described by time-varying digraph $\mathscr{G}(t)=\{\mathscr{V}, \mathscr{E}(t), \mathscr{A}(t)\}$, where $\mathscr{A}(t)=\left(a_{i j}(t)\right)_{N \times N}$ and the connections between agents and leader at time $t$ are described by $b_{i}(t), b_{i}(t)>0$ if and only if the agent $i$ can obtain information from the leader at time $t$; otherwise, $b_{i}(t)=0$.

In order to achieve leader-following consensus under switching topology, the impulsive control input is designed as

$$
\begin{aligned}
u_{i}(t)=-\sum_{k=1}^{+\infty} p_{1}[ & \left(\sum_{j \in N_{i}} l_{i j}(t)\left(x_{j}(t)-x_{i}(t)\right)\right. \\
& \left.\left.+b_{i}(t)\left(x_{i}(t)-x_{0}\right)\right)+p_{2} v_{i}\right] \delta\left(t-t_{k}\right),
\end{aligned}
$$


where $i=1,2, \ldots, N$. Let $\widetilde{\mathscr{G}}(t)=\{\widetilde{\mathscr{V}}, \widetilde{\mathscr{E}}(t), \widetilde{\mathscr{A}}(t)\}$ with $\widetilde{\mathscr{V}}(t)=$ $\{0,1,2, \ldots, N\}$ and

$$
\widetilde{\mathscr{A}}(t)=\left(\begin{array}{ccccc}
0 & 0 & 0 & \cdots & 0 \\
b_{1}(t) & 0 & a_{12}(t) & \cdots & a_{1 N}(t) \\
b_{2}(t) & a_{21}(t) & 0 & \cdots & a_{2 N}(t) \\
\vdots & \vdots & \vdots & \ddots & \vdots \\
b_{N}(t) & a_{N 1}(t) & a_{N 2}(t) & \cdots & 0
\end{array}\right) .
$$

Let $\widetilde{L}(t)=\left(\widetilde{l}_{i j}\right)_{(N+1) \times(N+1)}$ denotes the Laplace matrix of $\widetilde{\mathscr{G}}$. Equivalently, the multiagent system (2) with the impulsive controller (23) can be rewritten as follows:

$$
\begin{aligned}
& \dot{x}_{i}(t)=v_{i}(t), \quad \dot{v}_{i}(t)=0, \quad t \in\left(t_{k}, t_{k+1}\right], \\
& \Delta v_{i}\left(t_{k}\right)=-p_{1} \sum_{j \in \mathscr{V}} \tilde{l}_{i j}(t) x_{j}\left(t_{k}\right)-p_{2} v_{i}\left(t_{k}\right),
\end{aligned}
$$

where $i=0,1, \ldots, N$.

Remark 10. Note that the communication among agents only occurs at sampling times. This implies that interation graph does not contain any edges $\mathscr{G}(t)=0$ where $t \neq t_{k}$.

Similar to the discussion in Section 4, one has

$$
\begin{gathered}
x_{i}\left(t_{k+1}\right)=x_{i}\left(t_{k}\right)+h v_{i}\left(t_{k}^{+}\right), \\
v_{i}\left(t_{k+1}^{+}\right)=\left(1-p_{2}\right) v_{i}\left(t_{k}^{+}\right)-p_{1} \sum_{j=0}^{N} \widetilde{l}_{i j}\left(t_{k}\right) x_{j}\left(t_{k}\right) \\
-p_{1} h \sum_{j=0}^{N} \widetilde{l}_{i j}\left(t_{k}\right) v_{j}\left(t_{k}^{+}\right) .
\end{gathered}
$$

Let $\widetilde{x}_{i}(k)=x_{i}\left(t_{k}\right), \widetilde{v}_{i}(k)=x_{i}\left(t_{k}\right)+\alpha v_{i}\left(t_{k}^{+}\right)$, where $\alpha=2 h / p_{2}$.

It is easy to know that the network (2) achieves leaderfollowing consensus, if $\tilde{x}_{i}(k) \rightarrow \beta$ and $\widetilde{v}_{i}(k) \rightarrow \beta$, for some $\beta \in \mathbb{R}, i \in \widetilde{\mathscr{V}}$.

From (26), one has

$$
\begin{aligned}
\tilde{x}_{i}(k+1)= & \left(1-\frac{p_{2}}{2}\right) \tilde{x}_{i}(k)+\frac{p_{2}}{2} \widetilde{v}_{i}(k), \\
\widetilde{v}_{i}(k+1)= & \frac{p_{2}}{2} \widetilde{x}_{i}(k)+\left(1-\frac{p_{2}}{2}\right) \widetilde{v}_{i}(k) \\
& -\left(\frac{2}{p_{2}}-1\right) p_{1} h \sum_{j=0}^{N} l_{i j}\left(\tilde{x}_{j}\left(t_{k}\right)\right) \\
& -p_{1} h \sum_{j=0}^{N} l_{i j} \widetilde{v}_{j}(k) .
\end{aligned}
$$

Let $\widetilde{x}(k)=\left(\widetilde{x}_{0}^{T}(k), \widetilde{x}_{1}^{T}(k), \widetilde{x}_{2}^{T}(k), \ldots, \widetilde{x}_{N}^{T}(k)\right)^{T}$ and $\widetilde{v}(k)=$ $\left(\widetilde{v}_{0}^{T}(k), \widetilde{v}_{1}^{T}(k), \widetilde{v}_{2}^{T}(k), \ldots, \widetilde{v}_{N}^{T}(k)\right)^{T}$; then,

$$
\left(\begin{array}{l}
\tilde{x}(k+1) \\
\widetilde{v}(k+1)
\end{array}\right)=\mathbf{P}(k) \times\left(\begin{array}{l}
\tilde{x}(k) \\
\widetilde{v}(k)
\end{array}\right),
$$

where

$\mathbf{P}(k)$

$$
=\left(\begin{array}{cc}
\left(1-\frac{p_{2}}{2}\right) I & \frac{p_{2}}{2} I \\
\frac{p_{2}}{2} I-\left(\frac{2}{p_{2}}-1\right) p_{1} h \widetilde{L}(k) & \left(1-\frac{p_{2}}{2}\right) I-p_{1} h \widetilde{L}(k)
\end{array}\right) .
$$

Before moving on, the following lemmas are needed.

Lemma 11 (see [16]). Let $m \geq 2$ be a positive integer, and let $P_{1}, P_{2}, \ldots, P_{m}$ be non-negative $N \times N$ matrices with positive diagonal entries; then, $P_{1} P_{2} \cdots P_{m} \geq \varepsilon\left(P_{1}+P_{2}+\cdots+P_{m}\right)$, where $\varepsilon>0$ can be specified from matrices $P_{i}, i=1,2, \ldots, m$.

Lemma 12 (see [39]). Let $P_{1}, P_{2}, \ldots, P_{k} \in \mathbb{R}^{N \times N}$ be a finite set of SIA matrices with the property that for each sequence $P_{i_{1}}, P_{i_{2}}, \ldots, P_{i_{j}}$ of positive length, the matrix product $P_{i_{1}} P_{i_{2}} \cdots P_{i_{j}}$ is SIA. Then, for each infinite sequence $P_{i_{1}}, P_{i_{2}}, \ldots$, $P_{i_{i}}$, there exists a column vector $y$ such that

$$
\lim _{j \rightarrow \infty} P_{i_{1}} \times P_{i_{2}} \times \cdots P_{i_{j}}=\mathbf{1}_{N} y^{T} .
$$

Lemma 13 (see [40]). Suppose that $\mathbf{P} \in \mathbb{R}^{N \times N}$ is a row stochastic matrix with positive diagonal elements. If the digraph $\mathscr{G}(\mathbf{P})$ has a directed spanning tree, then $\mathbf{P}$ is SIA.

Lemma 14. Let

$$
\mathbf{P}=\left(\begin{array}{cc}
(1-\alpha) I & \alpha I \\
\beta I-\mu_{1} L & (1-\beta) I-\mu_{2} L
\end{array}\right)
$$

be non-negative matrix, where $\alpha, \mu_{1}, \mu_{2}>0$. If $L$ is a Laplace matrix of a digraph $\mathscr{G}$, which has a directed spanning tree, then $\mathbf{P}$ is a row stochastic matrix and the digraph of $\mathbf{P}$ contains a directed spanning tree.

Proof. It is easy to check the non-negative matrix $\mathbf{P} \mathbf{1}_{2 N}=$ $\mathbf{1}_{2 N}$. Then, $\mathbf{P}$ is a row stochastic matrix. Let $\mathscr{G}(\mathbf{P})$ denote the digraph of $\mathbf{P}$. Then, the Laplace matrix of $\mathscr{G}(\mathbf{P})$ is

$$
L(\mathbf{P})=\left(\begin{array}{cc}
\alpha I & -\alpha I \\
-\beta I+\mu_{1} L & \beta I+\mu_{2} L
\end{array}\right) .
$$

Let $\gamma_{i}, i=1,2, \ldots, N$ denote the eigenvalues of $L$.

Let $\lambda$ be an eigenvalue of matrix $\mathbf{P}$; then, one has

$$
\begin{aligned}
& \operatorname{det}\left(\begin{array}{cc}
\lambda I-\alpha I & \alpha I \\
\beta I-\mu_{1} L & \lambda I-\beta I-\mu_{2} L
\end{array}\right) \\
& =\prod_{i=1}^{N}\left((\lambda-\alpha)\left(\lambda-\beta-\mu_{2} \gamma_{i}\right)-\alpha\left(\beta-\mu_{1} \gamma_{i}\right)\right) .
\end{aligned}
$$

Let $Q(\lambda)=(\lambda-\alpha)\left(\lambda-\beta-\mu_{2} \gamma_{i}\right)-\alpha\left(\beta-\mu_{1} \gamma_{i}\right)$. Then,

$$
Q(0)=\alpha \mu_{1} \gamma_{i}+\alpha \mu_{2} \gamma_{i} \text {. }
$$

Therefore, from (34), $\lambda=0$ only if $\gamma_{i}=0$. 
When $\gamma_{i}=0$,

$$
Q(\lambda)=\lambda^{2}-\lambda(\alpha+\beta)
$$

Thus, when $\gamma_{i}(L)=0$, the solutions of $Q(\lambda)=0$ are $\lambda=0$ and $\lambda=\alpha+\beta$. On the other hand, if $\mathscr{G}$ contains a spanning tree, $L$ only has one simple eigenvalue equal to zero. Therefore, $L(\mathbf{P})$ only has one simple eigenvalue equal to zero, which implies that the digraph of $\mathbf{P}$ has a spanning tree. The proof is completed.

Theorem 15. If there exists a positive integer $l$, the union of $\widetilde{\mathscr{G}}\left(t_{k}\right)$ across $k \in\left[k_{0}, k_{0}+l\right]$ contains a directed spanning tree, for any non-negative integer $k_{0}$, and

$$
\begin{gathered}
h<\frac{p_{2}^{2}}{2 p_{1}\left(2-p_{2}\right) \Delta}, \quad p_{2} \leq 1, \\
h<\frac{2-p_{2}}{2 p_{1} \Delta}, \quad 1<p_{2}<2,
\end{gathered}
$$

where $\Delta=\max _{i \in \mathscr{V}, k \in \mathbb{N}}\left\{l_{i i}(k)+b_{i}(k)\right\}$; then, the multiagent system (2) achieves the leader-following consensus.

Proof. Let

$$
\mathbf{P}(k)=\left(\begin{array}{ll}
P_{11}(k) & P_{12}(k) \\
P_{21}(k) & P_{22}(k)
\end{array}\right),
$$

where $\mathbf{P}(k)$ is defined in (29). From (1), one has $l_{i j}<0$, for $i \neq j$. Then, the following statements are satisfied:

(i) $P_{11}(k)$ is nonnegative if and only if $p_{2}<2$;

(ii) $P_{12}(k)$ is nonnegative if and only if $p_{2}>0$;

(iii) $P_{21}(k)$ is nonnegative if and only if

$$
\frac{p_{2}}{2}-\left(\frac{2}{p_{2}}-1\right) p_{1} h\left(l_{i i}(k)+b_{i}(k)\right)>0, \quad \text { for } i \in \mathscr{V} \text {; }
$$

(iv) $P_{22}(k)$ is nonnegative if and only if

$$
\left(1-\frac{p_{2}}{2}\right)-p_{1} h\left(l_{i i}(k)+b_{i}(k)\right)>0, \quad \text { for } i \in \mathscr{V} .
$$

If $0<p_{2}<2$, then $2 / p_{2}-1>0$ and $1-p_{2} / 2>0$. Note that $l_{i j}+b_{i}(k) \geq 0$ and $p_{1}>0$. Then, the following four statements are satisfied when $p_{2}<2$.

(i) If $l_{i j}+b_{i}(k)=0$, then we have

$$
\begin{gathered}
\frac{p_{2}}{2}-\left(\frac{2}{p_{2}}-1\right) p_{1} h\left(l_{i i}(k)+b_{i}(k)\right)>0, \\
\left(1-\frac{p_{2}}{2}\right)-p_{1} h\left(l_{i i}(k)+b_{i}(k)\right)>0,
\end{gathered}
$$

(ii) If $l_{i j}+b_{i}(k)>0$, and

$$
h<\frac{p_{2} / 2}{\left(2 / p_{2}-1\right) p_{1}\left(l_{i i}(k)+b_{i}(k)\right)},
$$

then we have

$$
\frac{p_{2}}{2}-\left(\frac{2}{p_{2}}-1\right) p_{1} h\left(l_{i i}(k)+b_{i}(k)\right)>0,
$$

(iii) If $l_{i j}+b_{i}(k)>0$, and

$$
h<\frac{1-p_{2} / 2}{p_{1}\left(l_{i i}(k)+b_{i}(k)\right)},
$$

then we have

$$
\left(1-\frac{p_{2}}{2}\right)-p_{1} h\left(l_{i i}(k)+b_{i}(k)\right)>0 .
$$

According to the previous discussion, (38) is satisfied, if $p_{2}<$ 2 , and

$$
h<\frac{p_{2} / 2}{\left(2 / p_{2}-1\right) p_{1} \Delta} .
$$

Equation (39) is satisfied, if $p_{2}<2$, and

$$
h<\frac{1-p_{2} / 2}{p_{1} \Delta} \text {. }
$$

Note that

$$
\frac{p_{2} / 2}{\left(2 / p_{2}-1\right) p_{1} \Delta}-\frac{1-p_{2} / 2}{p_{1} \Delta}=\frac{2-2 / p_{2}}{\left(2 / p_{2}-1\right) p_{1} \Delta} .
$$

Then, (38) and (39) are satisfied if (36) holds. This implies that $\mathbf{P}_{11}(k), \mathbf{P}_{12}(k), \mathbf{P}_{21}(k)$, and $\mathbf{P}_{22}(k)$ are nonnegative. Then, $\mathbf{P}(k)$ is also nonnegative. Note that

$$
\mathbf{P}(k) \mathbf{1}_{2 N+2}=\left(\begin{array}{c}
\mathbf{1}_{N+1} \\
\mathbf{1}_{N+1}-\frac{2}{p_{2}} p_{1} h \widetilde{L}(k) \mathbf{1}_{N+1}
\end{array}\right),
$$

and $\widetilde{L}(k) \mathbf{1}_{N+1}=\mathbf{0}$. Then, $\mathbf{P}(k) \mathbf{1}_{2 N+2}=\mathbf{1}_{2 N+2}, \mathbf{P}(k)$ is a row stochastic matrix. Then, $\sum_{k=k_{0}}^{k_{0}+l} \mathbf{P}(k)$ and $\prod_{k=k_{0}}^{k_{0}+l} \mathbf{P}(k)$ are also a row stochastic matrix.

Note that

$$
\begin{aligned}
\sum_{k=k_{0}}^{k_{0}+l} \mathbf{P}(k) & \\
= & (l+1) \\
& \times\left(\begin{array}{cc}
\left(1-\frac{p_{2}}{2}\right) I & \frac{p_{2}}{2} I \\
\frac{p_{2}}{2} I-\left(\frac{2}{p_{2}}-1\right) p_{1} h \mathbf{S}_{k_{0}}^{l} & \left(1-\frac{p_{2}}{2}\right) I-p_{1} h \mathbf{S}_{k_{0}}^{l}
\end{array}\right),
\end{aligned}
$$

where $\mathbf{S}_{k_{0}}^{l}=(1 /(l+1)) \sum_{k=k_{0}}^{k_{0}+l} \widetilde{L}(k)$.

The union of $\mathscr{G}\left(t_{k}\right)$ across $k \in\left[k_{0}, k_{0}+l\right]$, for any nonnegative integer $k_{0}$ contains a directed spanning tree. This implies that the digraph with the Laplace matrix $\mathbf{S}_{k_{0}}^{l}$ also contains a directed spanning tree. 


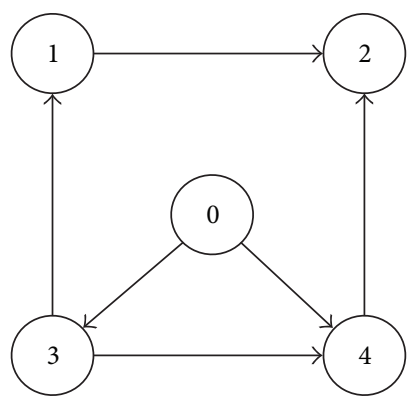

Figure 1: Fixed topology.

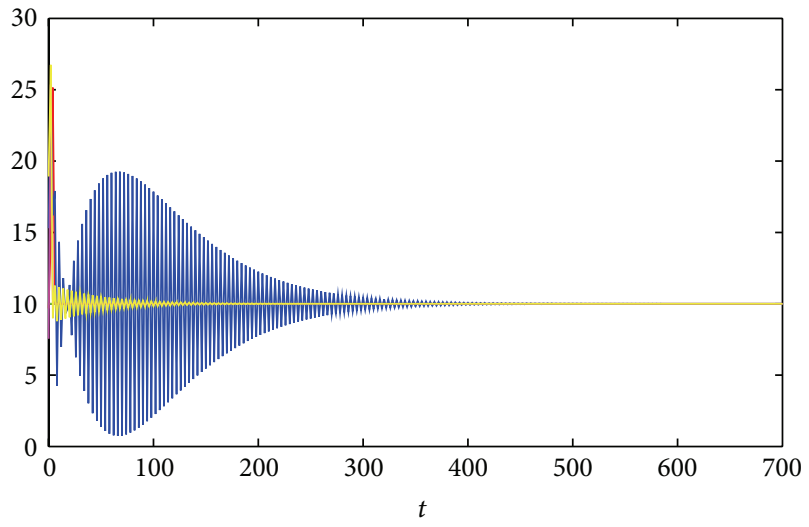

(a)

By Lemma 14, from (49), the digraph of $\sum_{k=k_{0}}^{k_{0}+l} \mathbf{P}(k)$ contains a spanning tree.

According to Lemma 11, one has

$$
\prod_{i=k_{0}}^{k_{0}+l} \mathbf{P}(i) \geq \varepsilon \sum_{i=k_{0}}^{k_{0}+l} \mathbf{P}(i)
$$

for some $\varepsilon$. This implies that the digraph of $\prod_{k=k_{0}}^{k_{0}+l} \mathbf{P}(k)$ also contains a spanning tree. It follows from Lemma 13 that $\prod_{k=k_{0}}^{k_{0}+l} \mathbf{P}(k)$ is SIA. By Lemma 12 ,

$$
\lim _{k \rightarrow \infty} \mathbf{P}(k) \mathbf{P}(k-1) \cdots \mathbf{P}(0) x(0)=\mathbf{1}_{2(N+1)} y^{T} x(0) .
$$

The proof is thus completed.

Remark 16. In this remark, we also show how to choose a suitable control gain $p_{1}$ and $p_{2}$ when the sampling period $h$ is given. According to Theorem 15, $p_{2}<2$ is also required. Similar to Remark 9 , one can choose $p_{2}$ from $(0,2]$, and then compute

$$
\Theta= \begin{cases}\frac{p_{2}^{2}}{2 h\left(2-p_{2}\right) \Delta}, & p_{2} \leq 1 \\ \frac{2-p_{2}}{2 h \Delta}, & 1<p_{2}<2 .\end{cases}
$$

Then, one can choose $p_{1}$ from $(0, \Theta)$.

\section{Illustrative Examples}

In this section, two illustrative numerical examples will be given to demonstrate the correctness of theoretical analysis.

5.1. Fix Topology. The communication topology is described as in Figure 1. The Laplacian matrix $L$ and matrix $B$ are given as follows:

$$
L=\left(\begin{array}{cccc}
1 & 0 & -1 & 0 \\
-1 & 2 & 0 & -1 \\
0 & 0 & 0 & 0 \\
0 & 0 & -1 & 1
\end{array}\right), \quad B=\operatorname{diag}(0,0,1,1)
$$

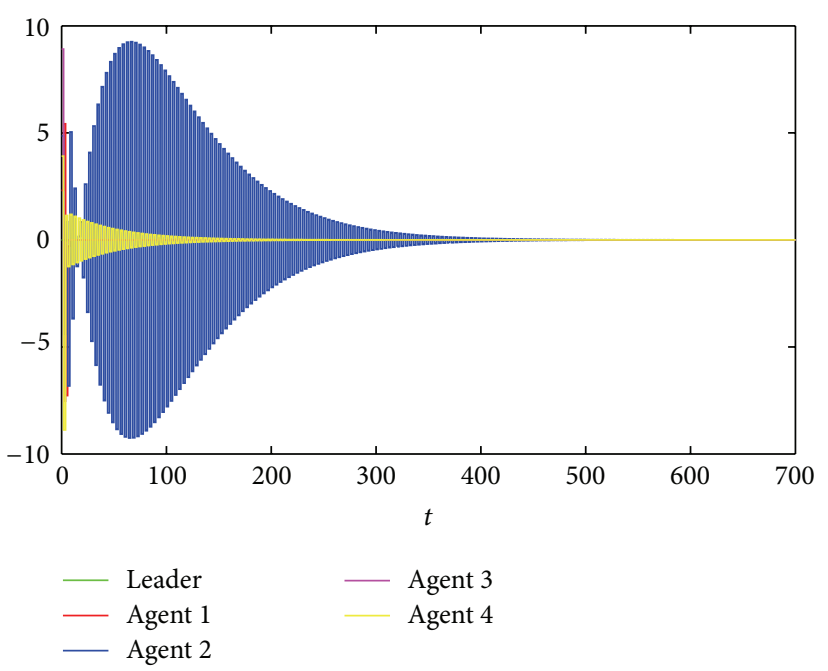

(b)

Figure 2: Trajectory of the multiagent system (2) under fixed topology, when $p_{1}=0.49$. Evolution of (a) $x_{i}$, and (b) $v_{i}$.

The eigenvalues of $L+B$ are $\lambda_{1}(L+B)=\lambda_{2}(L+B)=1$, $\lambda_{3}(L+B)=\lambda_{4}(L+B)=2$. Let $p_{2}=1, h=2$; according to Theorem 6 , the network can achieve leader-following consensus, if and only if

$$
p_{1}<\min _{i \in \mathscr{V}} \frac{2 p_{2}^{2}\left(2-p_{2}\right) \operatorname{Re}\left(\lambda_{i}\right)}{\left(\operatorname{Im}\left(\lambda_{i}\right)\left(p_{2}-2\right)\right)^{2} h+h p_{2}^{2} \operatorname{Re}^{2}\left(\lambda_{i}\right)}=0.5
$$

Figure 2 shows that the leader-following consensus can be achieved when $p_{1}=0.49$. But it cannot be achieved when $p_{1}=0.51$ (as shown in Figure 3).

5.2. Switching Topology. In this subsection, the network topology switches from a set $\left\{\widetilde{\mathscr{G}}_{1}, \widetilde{\mathscr{G}}_{2}, \widetilde{\mathscr{G}}_{3}, \widetilde{\mathscr{G}}_{4}\right\}$ as shown in 


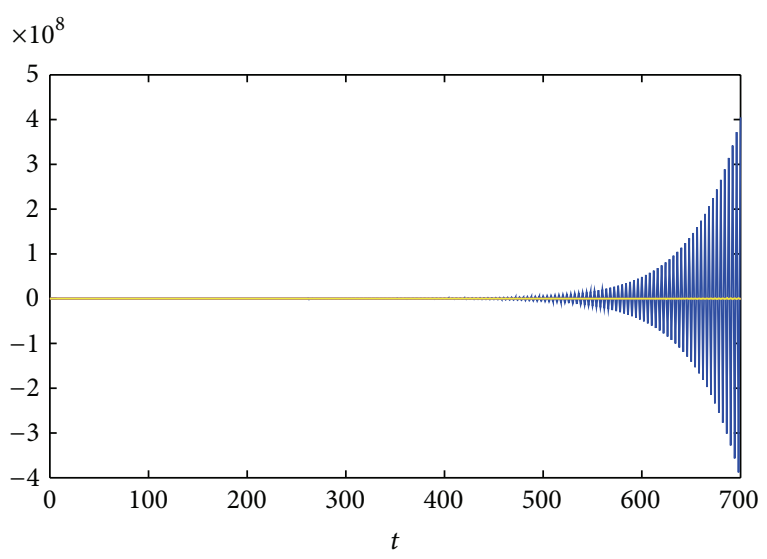

(a)
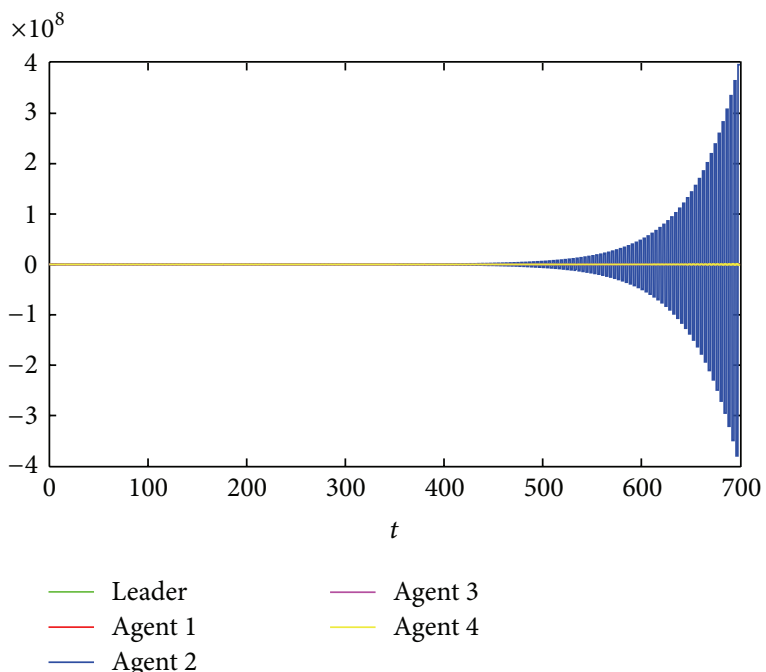

(b)

FIgURE 3: Trajectory of the multiagent system (2) under fixed topology, when $p_{1}=0.51$. Evolution of (a) $x_{i}$, and (b) $v_{i}$.

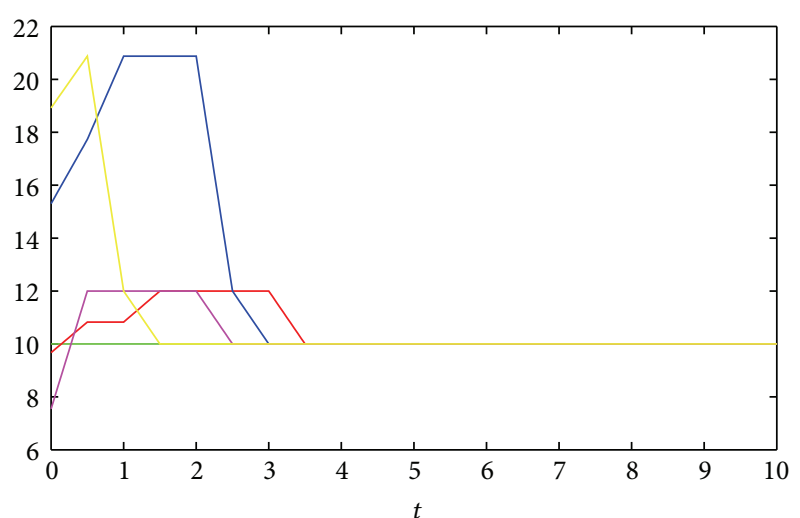

(a)

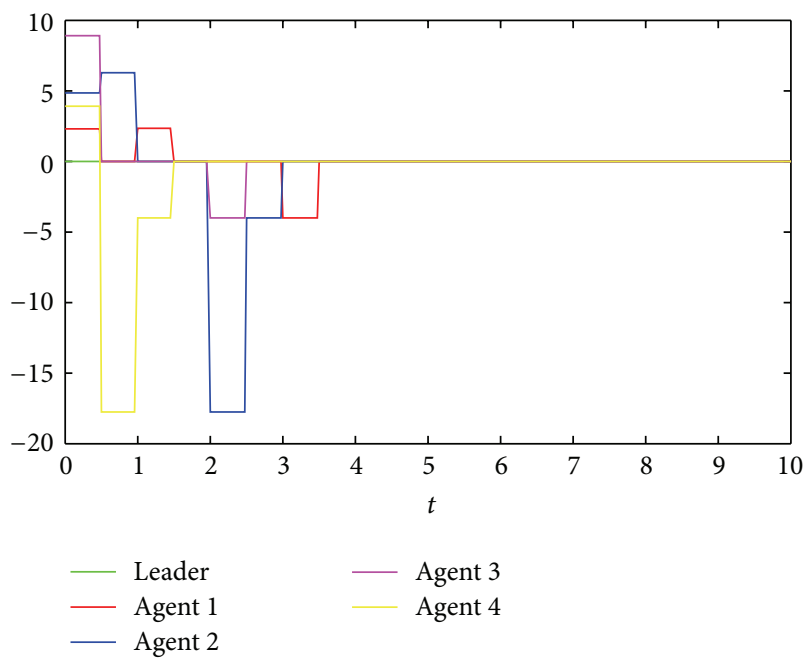

(b)

FIGURE 5: Trajectory of the multiagent system (2) under switched topology, when $p_{1}=0.95$. Evolution of (a) $x_{i}$, and (b) $v_{i}$.

Figure 4. The corresponding Laplacian matrices of $\mathscr{G}_{1}, \mathscr{G}_{2}$, $\mathscr{G}_{3}, \mathscr{G}_{4}$ and matrices $B_{1}, B_{2}, B_{3}, B_{4}$ are

$$
\begin{array}{rlrl}
L_{1} & =\left(\begin{array}{cccc}
0 & 0 & 0 & 0 \\
-1 & 1 & 0 & 0 \\
0 & 0 & 0 & 0 \\
0 & 0 & 0 & 0
\end{array}\right), & L_{2}=\left(\begin{array}{cccc}
0 & 0 & 0 & 0 \\
0 & 1 & 0 & -1 \\
0 & 0 & 0 & 0 \\
0 & 0 & -1 & 1
\end{array}\right), \\
L_{3}=\left(\begin{array}{cccc}
1 & 0 & -1 & 0 \\
0 & 0 & 0 & 0 \\
0 & 0 & 0 & 0 \\
0 & 0 & 0 & 0
\end{array}\right), & L_{4}=\left(\begin{array}{llll}
0 & 0 & 0 & 0 \\
0 & 0 & 0 & 0 \\
0 & 0 & 0 & 0 \\
0 & 0 & 0 & 0
\end{array}\right),
\end{array}
$$

$B_{1}=\operatorname{diag}\left(\begin{array}{llll}0 & 0 & 1 & 0\end{array}\right), B_{2}=\operatorname{diag}\left(\begin{array}{llll}0 & 0 & 0 & 0\end{array}\right), B_{3}=$ $\operatorname{diag}\left(\begin{array}{llll}0 & 0 & 0 & 1\end{array}\right)$, and $B_{4}=\operatorname{diag}\left(\begin{array}{llll}0 & 0 & 0 & 0\end{array}\right)$. Assume that $\mathscr{G}\left(t_{0}\right)=\mathscr{G}_{1}, \mathscr{G}\left(t_{1}\right)=\mathscr{G}_{2}, \mathscr{G}\left(t_{2}\right)=\mathscr{G}_{3}, \mathscr{G}\left(t_{3}\right)=\mathscr{G}_{4}, \mathscr{G}\left(t_{4}\right)=$ $\mathscr{G}_{1}, \ldots$ and $B\left(t_{0}\right)=B_{1}, B\left(t_{1}\right)=B_{2}, B\left(t_{2}\right)=B_{3}, B\left(t_{3}\right)=B_{4}$, $B\left(t_{4}\right)=B_{1}, \ldots$ Note that the union graph of $\widetilde{\mathscr{G}}_{1}, \widetilde{\mathscr{G}}_{2}, \widetilde{\mathscr{G}}_{3}$, and $\widetilde{\mathscr{G}}_{4}$, and $\max _{i \in \mathcal{N}, k \in \mathbb{N}}\left(l_{i i}(k)+b_{i}(k)\right)=1$. 
Let $p_{2}=1, h=0.5$, according to Theorem 15 , if

$$
p_{1}<\frac{p_{2}{ }^{2}}{2 h\left(2-p_{2}\right) \max _{i \in \mathcal{N}, k \in \mathbb{N}}\left\{l_{i i}(k)+b_{i}(k)\right\}}=1 .
$$

Figure 5 shows that the leader-following consensus can be achieved when $p_{1}=0.95$.

\section{Conclusions}

In this paper, the leader-following consensus problem of the multiagent system is considered. The impulsive control, which only needs sampled information and regulates the velocity of each agent at sampling times, is proposed for the leader-following consensus. Several new criteria are established for the leader-following consensus of the system under both fixed and switching topology. Illustrated examples have been given to show the effectiveness of the proposed impulsive control.

\section{Acknowledgment}

This work was supported in part by the National Natural Science Foundation of China under Grants 61073026, 61170031, 61272069 , and 61073025 .

\section{References}

[1] F. Bullo, J. Cortés, and S. Martínez, Distributed Control of Robotic Networks, Princeton Series in Applied Mathematics, Princeton University Press, Princeton, NJ, USA, 2009.

[2] Z.-H. Guan, Z.-W. Liu, G. Feng, and Y.-W. Wang, "Synchronization of complex dynamical networks with time-varying delays via impulsive distributed control," IEEE Transactions on Circuits and Systems. I, vol. 57, no. 8, pp. 2182-2195, 2010.

[3] R. Carli, A. Chiuso, L. Schenato, and S. Zampieri, "Distributed Kalman filtering based on consensus strategies," IEEE Journal on Selected Areas in Communications, vol. 26, no. 4, pp. 622633, 2008.

[4] A. Kashyap, T. Başar, and R. Srikant, "Quantized consensus," Automatica, vol. 43, no. 7, pp. 1192-1203, 2007.

[5] R. Olfati-Saber and R. M. Murray, "Consensus problems in networks of agents with switching topology and time-delays," IEEE Transactions on Automatic Control, vol. 49, no. 9, pp. 15201533, 2004.

[6] L. Moreau, "Stability of multiagent systems with time-dependent communication links," IEEE Transactions on Automatic Control, vol. 50, no. 2, pp. 169-182, 2005.

[7] F. Xiao and L. Wang, "Asynchronous consensus in continuoustime multi-agent systems with switching topology and timevarying delays," IEEE Transactions on Automatic Control, vol. 53, no. 8, pp. 1804-1816, 2008.

[8] Z. Lin, B. Francis, and M. Maggiore, "State agreement for continuous-time coupled nonlinear systems," SIAM Journal on Control and Optimization, vol. 46, no. 1, pp. 288-307, 2007.

[9] Q. Hui and W. M. Haddad, "Distributed nonlinear control algorithms for network consensus," Automatica, vol. 44, no. 9, pp. 2375-2381, 2008.

[10] Z.-W. Liu, Z.-H. Guan, T. Li, X.-H. Zhang, and J.-W. Xiao, "Quantized consensus of multi-agent systems via broadcast gossip algorithms," Asian Journal of Control, vol. 14, no. 6, pp. 1634-1642, 2012.

[11] T. Li and J.-F. Zhang, "Consensus conditions of multi-agent systems with time-varying topologies and stochastic communication noises," IEEE Transactions on Automatic Control, vol. 55, no. 9, pp. 2043-2057, 2010.

[12] P. Lin and Y. Jia, "Consensus of second-order discrete-time multi-agent systems with nonuniform time-delays and dynamically changing topologies," Automatica, vol. 45, no. 9, pp. 21542158, 2009.

[13] W. Yu, G. Chen, and M. Cao, "Some necessary and sufficient conditions for second-order consensus in multi-agent dynamical systems," Automatica, vol. 46, no. 6, pp. 1089-1095, 2010.

[14] Y. Hong, J. Hu, and L. Gao, "Tracking control for multiagent consensus with an active leader and variable topology," Automatica, vol. 42, no. 7, pp. 1177-1182, 2006.

[15] D. V. Dimarogonas, P. Tsiotras, and K. J. Kyriakopoulos, "Leader-follower cooperative attitude control of multiple rigid bodies," in Proceedings of the American Control Conference, pp. 801-806, Seattle, Wash, USA, 2008.

[16] A. Jadbabaie, J. Lin, and A. S. Morse, "Coordination of groups of mobile autonomous agents using nearest neighbor rules," IEEE Transactions on Automatic Control, vol. 48, no. 6, pp. 988-1001, 2003.

[17] Y. Cao and W. Ren, "Distributed coordinated tracking with reduced interaction via a variable structure approach," IEEE Transactions on Automatic Control, vol. 57, no. 1, pp. 33-48, 2012.

[18] H. Su, X. Wang, and Z. Lin, "Flocking of multi-agents with a virtual leader," IEEE Transactions on Automatic Control, vol. 54, no. 2, pp. 293-307, 2009.

[19] W. Zhu and D. Cheng, "Leader-following consensus of secondorder agents with multiple time-varying delays," Automatica, vol. 46, no. 12, pp. 1994-1999, 2010.

[20] Z. Meng, W. Ren, Y. Cao, and Z. You, "Leaderless and leaderfollowing consensus with communication and input delays under a directed network topology," IEEE Transactions on Systems, Man, and Cybernetics B, vol. 41, no. 1, pp. 75-88, 2011.

[21] T. Li and J. Zhang, "Sampled-data based average consensus with measurement noises: convergence analysis and uncertainty principle," Science in China F, vol. 52, no. 11, pp. 2089-2103, 2009.

[22] Y. Cao and W. Ren, "Sampled-data discrete-time coordination algorithms for double-integrator dynamics under dynamic directed interaction," International Journal of Control, vol. 83, no. 3, pp. 506-515, 2010.

[23] Y. Zhang and Y.-P. Tian, "Consensus of data-sampled multiagent systems with random communication delay and packet loss," IEEE Transactions on Automatic Control, vol. 55, no. 4, pp. 939-943, 2010

[24] H. Liu, G. Xie, and L. Wang, "Necessary and sufficient conditions for solving consensus problems of double-integrator dynamics via sampled control," International Journal of Robust and Nonlinear Control, vol. 20, no. 15, pp. 1706-1722, 2010.

[25] Y. Gao and L. Wang, "Consensus of multiple double-integrator agents with intermittent measurement," International Journal of Robust and Nonlinear Control, vol. 20, no. 10, pp.1140-1155, 2010.

[26] X. Liu and K. Rohlf, "Impulsive control of a Lotka-Volterra system," IMA Journal of Mathematical Control and Information, vol. 15, no. 3, pp. 269-284, 1998.

[27] J. E. Prussing and J.-H. Chiu, "Optimal multiple-impulse timefixed rendezvous between circular orbits," Journal of Guidance, Control, and Dynamics, vol. 9, no. 1, pp. 17-22, 1986. 
[28] J. F. Eastham and K. J. Hastings, "Optimal impulse control of portfolios," Mathematics of Operations Research, vol. 13, no. 4, pp. 588-605, 1988.

[29] G. Zhang, Z. Liu, and Z. Ma, "Synchronization of complex dynamical networks via impulsive control," Chaos, vol. 17, no. 4, Article ID 043126, 2007.

[30] B. Liu, X. Liu, G. Chen, and H. Wang, "Robust impulsive synchronization of uncertain dynamical networks," IEEE Transactions on Circuits and Systems. I, vol. 52, no. 7, pp. 1431-1441, 2005.

[31] J. Lu, D. W. C. Ho, and J. Cao, "A unified synchronization criterion for impulsive dynamical networks," Automatica, vol. 46, no. 7, pp. 1215-1221, 2010.

[32] Q. Zhang, S. Chen, and C. Yu, "Impulsive consensus problem of second-order multi-agent systems with switching topologies," Communications in Nonlinear Science and Numerical Simulation, vol. 17, no. 1, pp. 9-16, 2012.

[33] Y. Qian, X. Wu, J. Lu, and J.-A. Lu, "Second-order consensus of multi-agent systems with nonlinear dynamics via impulsive control," Neurocomputing, 2013.

[34] Z.-H. Guan, Z.-W. Liu, G. Feng, and M. Jian, "Impulsive consensus algorithms for second-order multi-agent networks with sampled information," Automatica, vol. 48, no. 7, pp. 13971404, 2012.

[35] Z.-W. Liu, Z.-H. Guan, X. Shen, and G. Feng, "Consensus of multi-agent networks with aperiodic sampled communication via impulsive algorithms using position-only measurements," IEEE Transactions on Automatic Control, vol. 57, no. 10, pp. 2639-2643, 2012.

[36] K. Ogata, Discrete-Time Control Systems, Prentice-Hall, Englewood Cliffs, NJ, USA, 2nd edition, 1995.

[37] P. Parks and V. Hahn, Stability Theory, Prentice Hall, Englewood Cliffs, NJ, USA, 1993.

[38] W. Yu, W. X. Zheng, G. Chen, W. Ren, and J. Cao, "Second-order consensus in multi-agent dynamical systems with sampled position data," Automatica, vol. 47, no. 7, pp. 1496-1503, 2011.

[39] J. Wolfowitz, "Products of indecomposable, aperiodic, stochastic matrices," Proceedings of the American Mathematical Society, vol. 14, pp. 733-737, 1963.

[40] W. Ren and R. W. Beard, "Consensus seeking in multiagent systems under dynamically changing interaction topologies," IEEE Transactions on Automatic Control, vol. 50, no. 5, pp. 655661, 2005. 


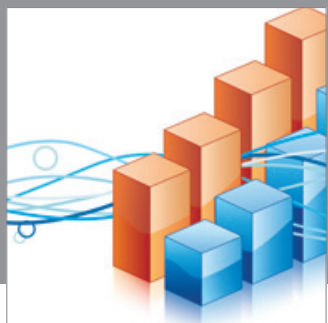

Advances in

Operations Research

mansans

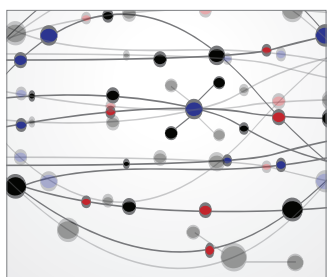

The Scientific World Journal
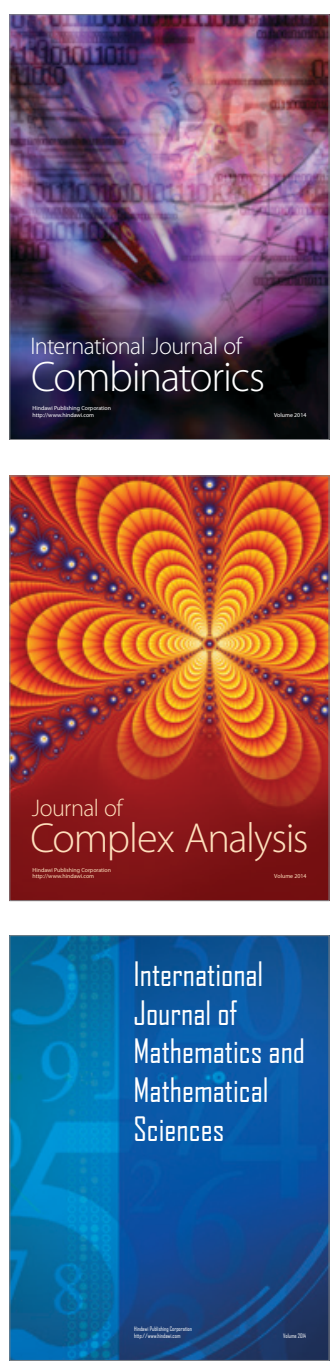
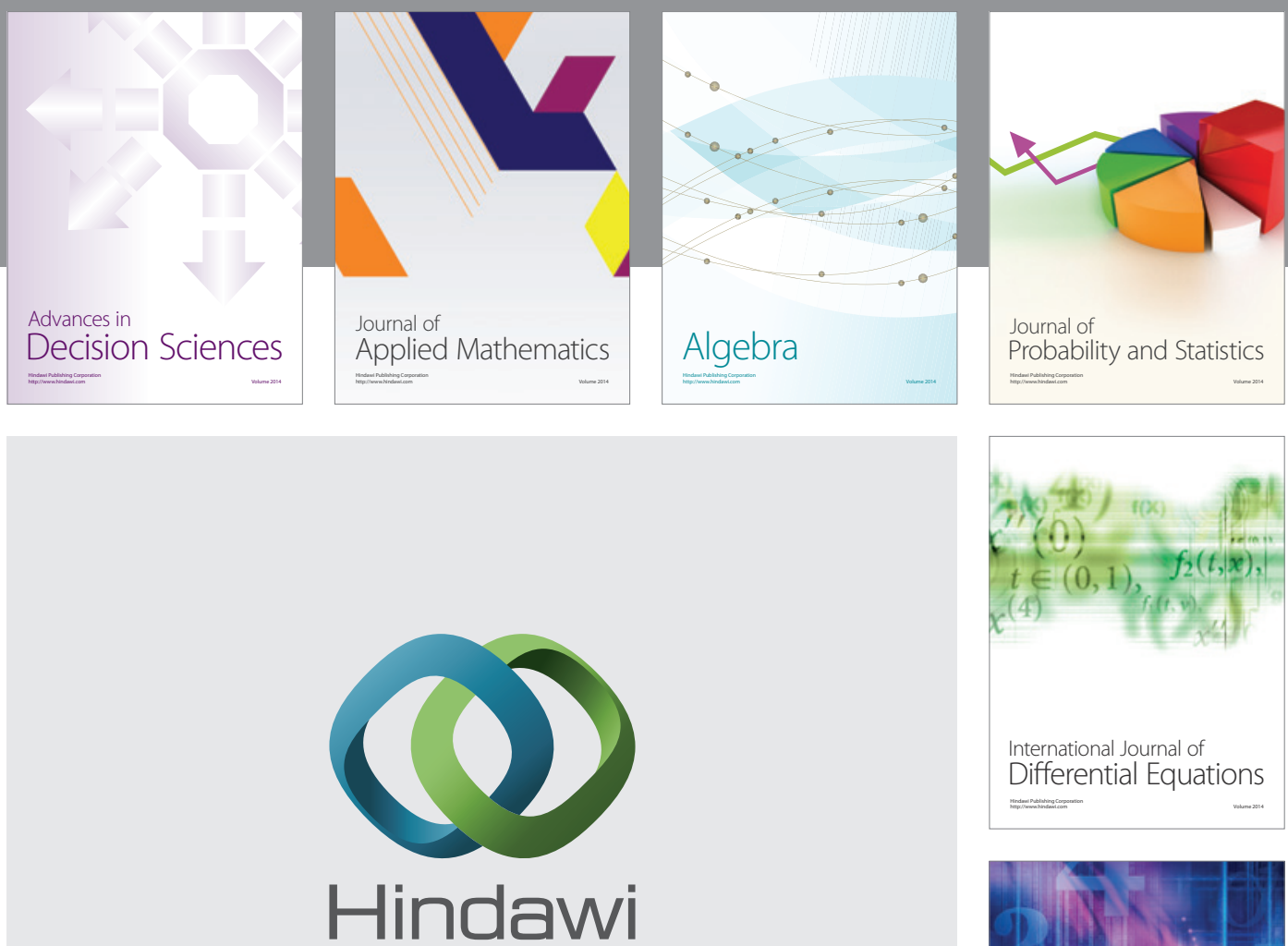

Submit your manuscripts at http://www.hindawi.com
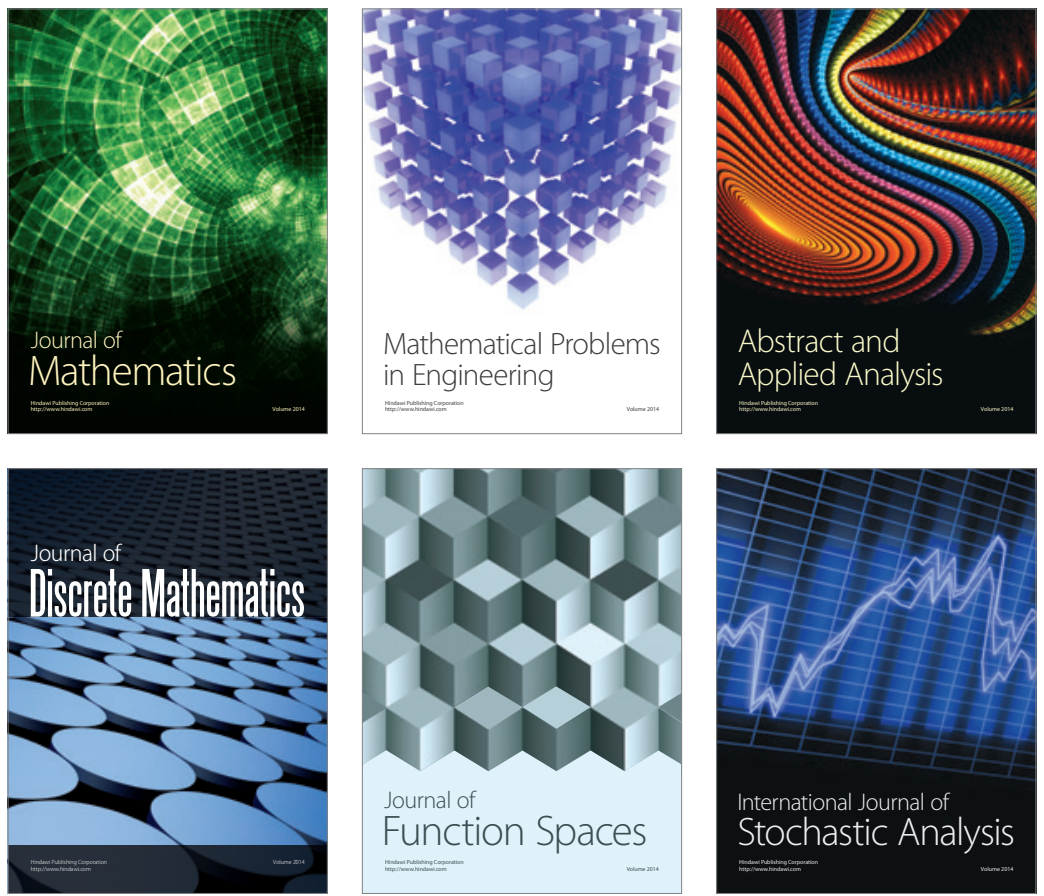

Journal of

Function Spaces

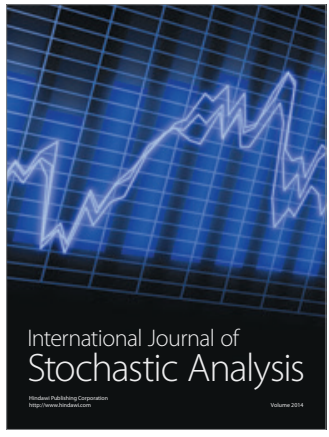

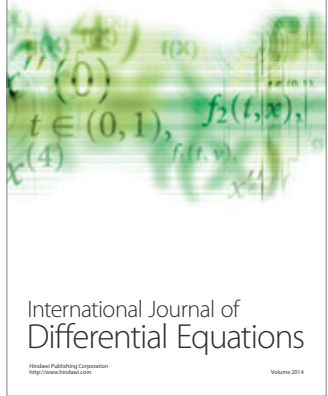
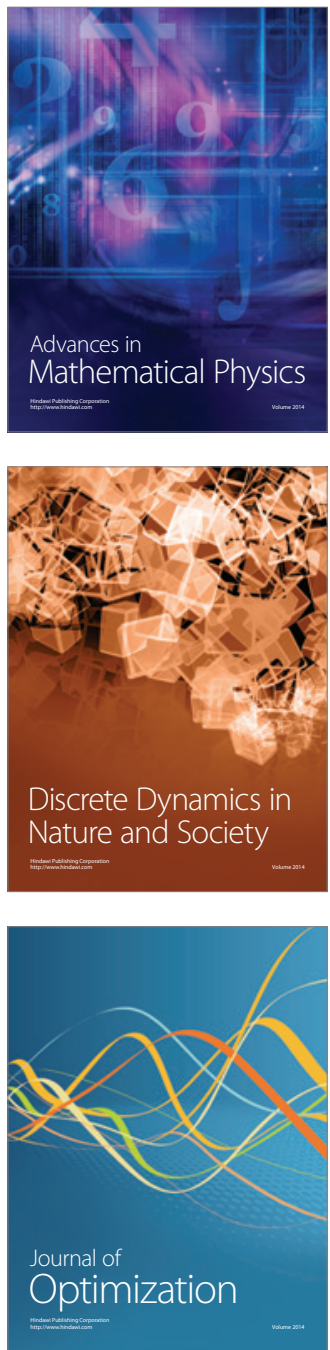